\title{
Metronidazole Induced Encephalopathy: A Case Report Showing Devastating Course on Consecutive MR Imaging
}

\author{
Soo Yeon Jeong ${ }^{1}$, Se Jeong Jeon ${ }^{1,}{ }^{*}$, Youe Ree Kim ${ }^{1}$ and See Sung Choi ${ }^{1}$ \\ ${ }^{1}$ Department of Radiology, Wonkwang University College of Medicine and Hospital, Muwang-Ro, Iksan, Jeonlabuk-do, Republic of Korea \\ "Corresponding author: Se Jeong Jeon, Department of Radiology, Wonkwang University College of Medicine and Hospital, 895 Muwang-Ro, Iksan, Jeonlabuk-do, 54538, \\ Republic of Korea. Tel: +82-638591920, Fax: +82-638514749, E-mail: medicalq@hanmail.net
}

Received 2018 February 07; Revised 2018 March 27; Accepted 2018 May 20.

\begin{abstract}
Metronidazole-induced encephalopathy is a rare neurologic adverse effect of an antibiotic drug 'metronidazole'. Although it is usually reversible with immediate cessation of the drug, it can also be fatal when used continuously. Here, we present a 72- yearold female who was on metronidazole for a total of 46 consecutive days. Her initial MRI on the 22nd day of medication use revealed a typical involvement of the cerebellar dentate nuclei, corpus callosum splenium, and periaqueductal midbrain, suggesting metronidazole-induced encephalopathy. However, she continued the medication for another 24 days, until she revisited our emergency department with mental status change. Her consecutive MRI showed diffuse cerebral and brainstem involvement, as well as a more prominent involvement of the dentate nuclei and splenium. We present this case to highlight that it is possible for metronidazole-induced toxic encephalopathy to worsen without immediate withdrawal of the offending drug.
\end{abstract}

Keywords: Metronidazole, Toxic Encephalopathy, Neurotoxicity

\section{Introduction}

Metronidazole is a commonly prescribed 5nitroimidazole group antibiotic, which is used for various anaerobic bacterial and protozoan infections; however, it may also be used to manage hepatic encephalopathy or Crohn's disease. It is considered relatively safe, but may produce a number of neurological adverse effects, such as ataxia, seizures, peripheral neuropathy, and, albeit much less commonly, encephalopathy (1). There already have been several reports of metronidazole-induced encephalopathy (MIE) depicting its characteristic findings on MR imaging. However, to the best of our knowledge, only a few of these reports described the course of MIE on follow-up MR imaging resulted from the continuous use of the medication. Here, we present a case of MIE, which remained uncorrected for a while, and ultimately resulted in a devastating course depicted on a follow-up MR imaging.

\section{Case Presentation}

A 72-year-old woman with a history of advanced liver cirrhosis presented to our emergency department with chief complaints of dizziness and dysarthria for one day. Her family denied any alcohol intake, nor any history of traumatic insult. On presentation, she showed severe dysarthria; however, neurologically her sensory and motor functions were intact. Cerebellar function test was not performed due to low patient compliance. All other neurological examinations showed almost normal results. Her initial lab findings showed slightly increased level of glucose (113 mg/dL; normal range $70-100 \mathrm{mg} / \mathrm{dL}$ ) and normal level of ammonia ( $24 \mathrm{uM} / \mathrm{L}$; normal range 11 - $51 \mathrm{u} \mathrm{M} / \mathrm{L}$ ). Liver function test showed mildly increased aspartate aminotransferase (AST) level (84 IU/L; normal range 5 - 35 IU/L). Non-contrast brain CT image did not show any abnormality (not shown). Meanwhile, clinicians noticed that she was on flasinyl (brand name for drug metronidazole) $500 \mathrm{mg}$ orally TID for spontaneous bacterial peritonitis, making a cumulative dose of $33 \mathrm{~g}$ for a duration of 22 days. She underwent further evaluation via MRI. MR images demonstrated symmetrical high signal intensities involving the cerebellar dentate nuclei, corpus callosum splenium, and periaqueductal midbrain on fluid-attenuated inversion recovery(FLAIR) and T2-weighted images. On diffusion weighted imaging (DWI), corpus callosum splenium and periaqueductal midbrain lesions showed corresponding high sig- 
nal intensity. However, on apparent diffusion coefficient (ADC) map, only splenial lesion showed a low ADC value, suggesting cytotoxic edema (Figure 1A-E). Possible diagnosis of MIE was made based on MRI findings and clinical history. Clinicians recommended immediate cessation of the drug, but she was discharged and disregarded the advice, continuing the use of the drug for an additional 24 days, until she revisited our emergency department, presenting aggravated dysarthria and drowsy mental status. Followup brain MR imaging demonstrated diffuse hyperintensities on the entire cerebral white matter on FLAIR and T2weighted images. Moreover, there were diffuse high signal intensity lesions along brainstem. The cerebellar dentate nuclei showed greater prominence in hyperintensity compared with the initial FLAIR and T2-weighted images, together along suggesting the worse progression of the MIE (Figure 2A-C). Hence, the patient discontinued metronidazole and went on conservative treatment. Her mental status seemed to gradually improve so that she could somewhat obey to a command on the 16th day of admission. However, further follow-up imaging was not possible due to patient's poor general condition and family's disagreement to additional MR imaging. Regardless of the neurological improvement, she had other comorbid conditions at the time of admission and she expired due to kidney failure and aggravated metabolic acidosis after an additional one month.

\section{Discussion}

Metronidazole is a synthetic nitroimidazole group antibiotic that is originally introduced to treat Trichomonas vaginalis; however, is now widely used to treat anaerobic bacterial and protozoal infections (2). In our case, metronidazole was used to both treat and prevent spontaneous bacterial peritonitis. The drug can cross the blood-brainbarrier (BBB) and reach a high concentration in the central nervous system, showing $60 \%-100 \%$ of plasma concentration in the nervous system $(3,4)$. For this reason, metronidazole can be used as the treatment for central nervous system (CNS) infection, such as brain abscess. However, for the same reason, it may produce several neurological adverse effects including encephalopathy (2). Since 1977, the year in which the first case report of MIE was published, several cases have been reported but the exact mechanism of MIE remained unclear. However, it is hypothesized that a toxic radical induced by the oxygenation of metronidazole derivatives might cause potentially reversible axonal swelling $(5,6)$. Vascular spasms may also play a role by producing reversible localized ischemia (2). Modulation of the gamma-amino butyric acid (GABA) receptors within the cerebellar and vestibular systems has also been proposed as possible mechanisms $(4,5)$.

To diagnose MIE, neuroimaging findings are most important. The characteristic MR imaging findings are best visualized with T2-weighted or FLAIR sequence images as bilateral symmetric high signal intensity lesions. Axonal swelling with increased water content or localized vascular ischemia due to vasospasm may cause the signal change (7). Typical locations of involvement are cerebellar dentate nuclei, midbrain (tectum, red nucleus, and tegmentum around periaqueductal gray matter), dorsal pons and medulla, and corpus callosum splenium in decreasing order of frequency. Among these, the cerebellar dentate nuclei are involved in most cases and considered characteristic for MIE. Uncommon locations, such as subcortical white matter, inferior colliculus, basal ganglia, thalamus, and middle cerebellar peduncle, may also be involved (2).

In our presented case, initially, there were hyperintensities in cerebellar dentate nuclei, corpus callosum splenium, and periaqueductal midbrain on FLAIR and T2 images, which are quite typical locations indicating the involvement of MIE. Together with clinical history, the diagnosis of MIE could be made. After unintended prolonged use of the drug for another 24 days, MR images of the same patient showed diffuse involvement of both cerebral hemispheres and brainstem, along with more prominently high signal intensities at the initial involvement sites.

Cho et al. previously reported that brain MRI findings can show temporal progression according to the interval between symptom onset and MR imaging. As claimed by this report, the splenium was initially involved, the brainstem and dentate nuclei were usually involved within 1 week after administration, and diffuse subcortical white matter involvement was the last presentation in patients with prolonged administration (6). MR images of our case after 22 days of medication showed T2 hyperintensities at the splenium, periaqueductal area, and dentate nuclei; moreover, the follow up MR images after total 46 days also demonstrated diffuse subcortical white matter involvements, which were consistent with previous study.

The only definitive treatment for MIE is withholding the drug as early as possible. In most cases, patients' symptoms as well as MRI findings can be improved following immediate drug discontinuation. According to a previously published report, most patients recovered between 3 and 

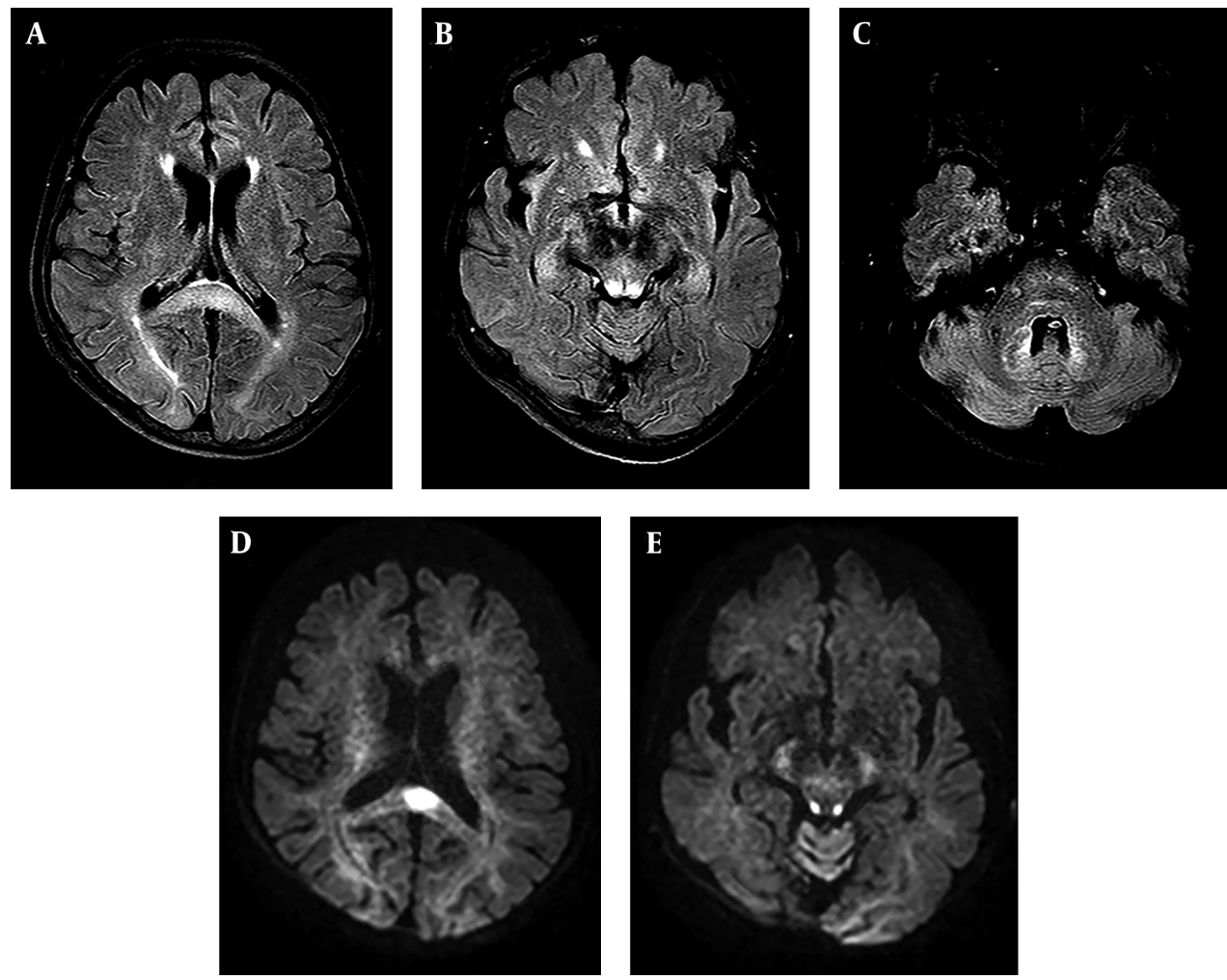

Figure 1. A 72-year-old woman with a history of advanced liver cirrhosis presented with dizziness and dysarthria. MR fluid-attenuated inversion recovery (FLAIR) (A - C) and diffusion weighted imaging (DWI) (D and E) images taken on the 22nd day of metronidazole use demonstrate typical involvement sites of metronidazole-induced encephalopathy. On FLAIR images, corpus callosum splenium (A), periaqueductal midbrain (B), and cerebellar dentate nuclei (C) shows high signal intensities. Among these, corpus callosum splenium (D) and periaqueductal midbrain (E) shows corresponding high signal intensities on DWI. Only corpus callosum showed low apparent diffusion coefficient (ADC) value, suggesting cytotoxic edema in this area (not shown).
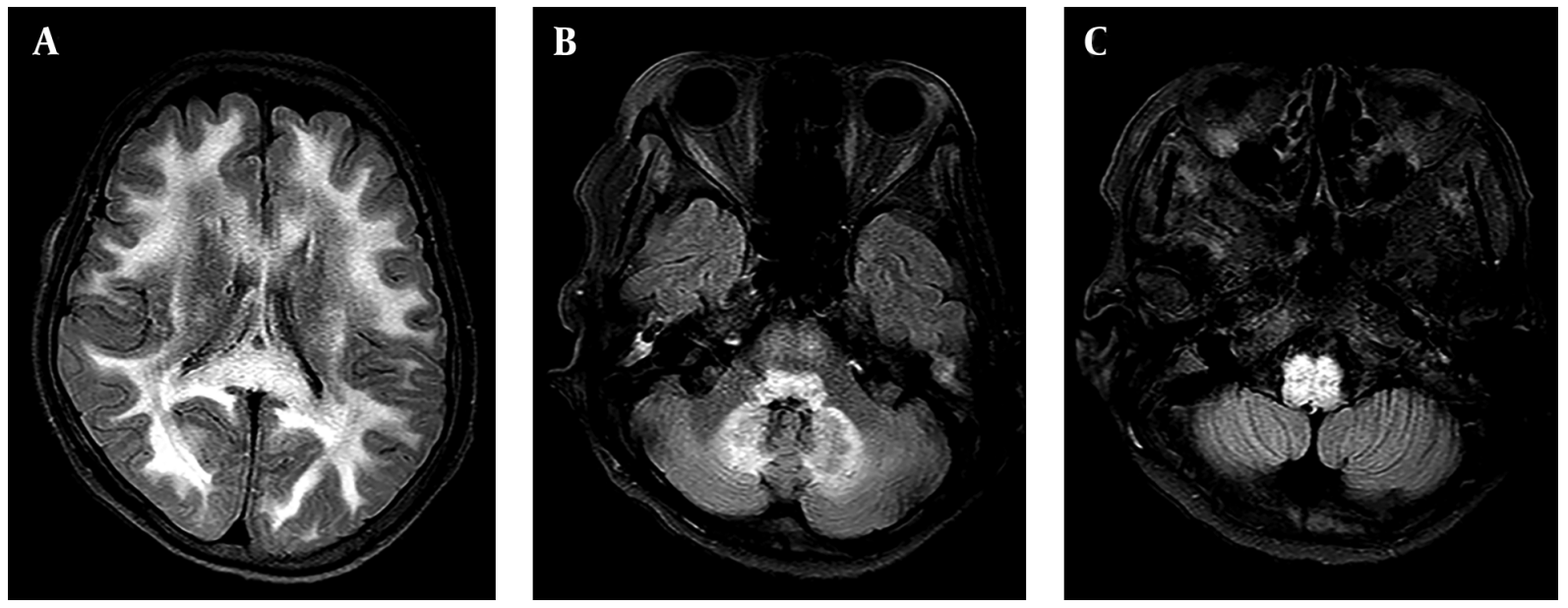

Figure 2. A - C, Consecutive MR fluid-attenuated inversion recovery (FLAIR) images taken on total 46th day of continuous metronidazole use demonstrate the worse progression of the toxic encephalopathy. Diffuse high signal intensities are shown in both cerebral white matter (A), cerebellar dentate nuclei (B), and along the brainstem (C).

16 weeks after cessation with resolution of abnormal MRI lesions (8). However, MIE is not always reversible and can be a potential cause of death. Hobbs et al. demonstrated that persistent encephalopathy with poor outcome may 
occur and that the duration between symptom onset and cessation of the drug may be important prognostic factors (9). Although the cause of death in our patient was possibly due to acute kidney failure and metabolic acidosis, our case also highlights the fact that it can be devastating if the medication is not withheld immediately.

In conclusion, MIE is an uncommon adverse effect of metronidazole treatment which can not only involve the dentate nuclei, but also the corpus callosum and brainstem on brain MR imaging. Generally, MIE is considered reversible with cessation of the drug. However, with prolonged use of the drug, as presented in this case report, MR imaging can show devastating progression, showing diffuse cerebral involvement. Clinicians and radiologists should be aware of the potentially fatal adverse effects of this generally well-tolerated drug.

\section{Footnotes}

Authors' Contributions: None declared.

Financial Disclosure: None declared.

Funding/Support: None declared.

\section{References}

1. Kumari P, Porwal YC, Arora A, Kumar M, Kumar D. Metronidazole induced neurotoxicity in a case of liver abscess. Int J Hepatobiliary Pancreat Dis. 2016;6(96-9). doi: 10.5348/ijhpd-2016-61-CR-17.
2. Roy U, Panwar A, Pandit A, Das SK, Joshi B. Clinical and Neuroradiological Spectrum of Metronidazole Induced Encephalopathy: Our Experience and the Review of Literature. J Clin Diagn Res. 2016;10(6):OE019. doi: 10.7860/JCDR/2016/19032.8054. [PubMed: 27504340]. [PubMed Central: PMC4963700].

3. Lamp KC, Freeman CD, Klutman NE, Lacy MK. Pharmacokinetics and pharmacodynamics of the nitroimidazole antimicrobials. Clin Pharmacokinet. 1999;36(5):353-73. doi: 10.2165/00003088-19993605000004. [PubMed: 10384859].

4. Knorr JP, Javed I, Sahni N, Cankurtaran CZ, Ortiz JA. Metronidazoleinduced encephalopathy in a patient with end-stage liver disease. Case Reports Hepatol. 2012;2012:209258. doi: 10.1155/2012/209258. [PubMed: 25374704]. [PubMed Central: PMC4208391].

5. Kim E, Na DG, Kim EY, Kim JH, Son KR, Chang KH. MR imaging of metronidazole-induced encephalopathy: lesion distribution and diffusion-weighted imaging findings. AJNR Am J Neuroradiol. 2007;28(9):1652-8. doi:10.3174/ajnr.A0655. [PubMed: 17885234].

6. Cho J, Lee SJ. Metronidazole-induced encephalopathy diagnosed by follow-up brain magnetic resonance imaging. J Neurocrit Care. 2016;9(1):16-20. doi: 10.18700/jnc.2016.9.1.16.

7. Singh R, Kaur R, Pokhariyal P, Aggarwal R. Sequential MR imaging (with diffusion-weighted imaging) changes in metronidazoleinduced encephalopathy. Indian J Radiol Imaging. 2017;27(2):129-32. doi: 10.4103/ijri.IJRI_341_16. [PubMed: 28744071]. [PubMed Central: PMC5510308].

8. Thakkar N, Bhaarat, Chand R, Sharma R, Mahavar S, Srivastava S, et al. Metronidazole Induced Encephalopathy. J Assoc Physicians India. 2016;64(11):72-4. [PubMed: 27805338].

9. Hobbs K, Stern-Nezer S, Buckwalter MS, Fischbein N, Finley Caulfield A. Metronidazole-induced encephalopathy: not always a reversible situation. Neurocrit Care. 2015;22(3):429-36. doi: 10.1007/s12028-014-01029. [PubMed: 25561434]. 